\title{
Treatment with anti-SARS-CoV-2 monoclonal antibodies in pregnant and postpartum women: first experiences in Florence, Italy
}

\author{
Tommaso Manciulli ${ }^{1}$ Giulia Modi ${ }^{1}$ Irene Campolmi ${ }^{2,3} \cdot$ Beatrice Borchi $^{2,3} \cdot$ Michele Trotta $^{2,3} \cdot$ Michele Spinicci $^{1,2}$. \\ Filippo Lagi ${ }^{1,2} \cdot$ Alessandro Bartoloni $^{1,2} \cdot$ Lorenzo Zammarchi $^{1,2,3}$ (i)
}

Received: 15 November 2021 / Accepted: 5 February 2022 / Published online: 7 March 2022

(c) The Author(s) 2022, corrected publication 2022

\begin{abstract}
Purpose Pregnant and postpartum women are at increased risk of developing severe COVID-19. Monoclonal antibodies (mAbs) are now widely used in high-income countries to treat mild to moderate COVID-19 outpatients at risk for developing severe disease. Very few data are available on the use of mAbs in special populations, including pregnant and postpartum women. Here we present our early experience with mAbs in these two populations.

Methods Electronic records of pregnant and postpartum women treated with mAbs at Careggi University Hospital, Florence, were retrieved. Relevant data were extracted (age, presence of risk factors for COVID-19, oxygen support, mAb type, gestational age, and pregnancy status). When available, outcomes at 28 days after administration were also included.

Results From March 1st to September 30th 2021, eight pregnant and two postpartum women have been treated with mAbs at our center. The median age was 31 years (IQR 30-33.5, range 29-38), median gestational age was 24 weeks. Seven patients had additional risk factors. According to the Italian disposition, all patients received casirivimab/imdevimab, with five receiving a $2.4 \mathrm{mg}$ dose and five receiving a $8 \mathrm{~g}$ dose. Eight patients improved. One developed myocarditis, considered a COVID-19 complication. Another required a transient increase of low flow oxygen support before improving and being discharged. At a 28 days follow-up, all patients were clinically recovered. We did not observe mAbs related adverse events. Conclusion Although preliminary data should be interpreted with caution, it is remarkable how mAbs were well tolerated by pregnant women with COVID-19. Further data on mAbs in this special population should be collected but the use of mAbs in pregnant and postpartum patients should be considered. Even thus oral antivirals are becoming available, they are not recommended in pregnant and postpartum women. This population may specifically benefit from treatment with last generation mAbs.
\end{abstract}

Keywords SARS-CoV-2 $\cdot$ Pregnancy $\cdot \mathrm{mAbs} \cdot$ Monoclonal antibodies $\cdot$ Casirivimab/imdevimab $\cdot$ COVID-19

\section{Background}

For about 2 years, the world has dealt with the severe acute respiratory syndrome coronavirus (SARS-CoV)-2 pandemic. While it was immediately clear that older adults

Lorenzo Zammarchi

lorenzo.zammarchi@unifi.it

1 Department of Experimental and Clinical Medicine, University of Florence, Largo Brambilla 3, Largo Giovanni Alessandro Brambilla, 3, 50134 Florence, FI, Italy

2 Infectious and Tropical Diseases Unit, Careggi University Hospital, Florence, Italy

3 Referral Centre for Infectious Diseases in Pregnancy, Careggi University Hospital, Florence, Italy with comorbidities had an increased risk for mortality and severe disease manifestations [1-3], pregnant and postpartum women were later recognized to be at higher risk for developing severe disease and poor outcomes [4-6] especially if the infection occurs in the third trimester [7]. In particular, a systematic review found that pregnant women have a higher odds of death (2.58) and of ICU admission (18.58) and babies born from pregnant COVID-19 patients also have a higher odd for neonatal ICU admission (4.58) [6]. The Centers for Disease Control and Prevention (CDC) listed pregnancy among the medical conditions associated with higher risk for severe COVID-19 [3].

Pregnant women were initially excluded from COVID19 vaccination campaigns due to the absence of safety and efficacy data on this subset of patients [8,9]. However, since 
mid-2021, several scientific societies have recommended vaccination of pregnant women against COVID-19 [8]. The relative delay and the fact that some pregnant women may feel hesitant about being vaccinated, put many pregnant women at risk for being infected with SARS-CoV-2.

From March 2021, monoclonal antibodies (mAbs) became available to treat COVID-19 in Italy [10]. At first, these were used only in outpatients with mild to moderate COVID-19, with risk factors for developing severe disease, and within 10 days from symptoms onset [11]. Then, recent data has shown a potential benefit in inpatients hospitalized for COVID-19 [12]. For this reason, since August 2021, in Italy the Agenzia Italiana del Farmaco (AIFA) has authorized the use of a higher dose of mAbs (casirivimab/ imdevimab $8 \mathrm{~g}$ ) in hospitalized seronegative patients with COVID-19, not requiring high flow oxygen or mechanical ventilation [13]. Likewise, the National Health Institute (NIH) guidelines state that the therapeutic management of pregnant patients with COVID-19 should be the same as for nonpregnant patients and anti-SARS-CoV-2 mAbs can be considered for pregnant people with COVID-19, especially those who have additional risk factors for severe disease [14].

AIFA includes "primary and secondary immunosuppression conditions" as risk factors for developing severe COVID-19 and eligibility criteria for mAbs prescription in outpatient subjects [11]. Considered the immune alterations associated with pregnancy and puerperium [15], the increasing evidence on poorer COVID-19 outcome in pregnant women $[9,16-21]$ and the recommendations issued by the National Institute of Health (NIH) [14] as well as the Royal College of Obstetrics and Gynecologists [22], in our center we offered mAbs to all pregnant women with mild to moderate COVID-19, not requiring hospitalization. Nevertheless, the use of mAbs in pregnant women is still scarcely documented in the medical literature. Here, we report the early results on the use of $\mathrm{mAb}$ to treat pregnant or postpartum patients at a single center in Italy.

\section{Methods}

\section{Inclusion criteria}

Electronic records of pregnant patients treated with mAbs from March 1st 2020 to September 30th 2021 at the Infectious and Tropical Diseases Unit, Careggi University Hospital, Florence, Italy, were retrieved. We included any pregnant or postpartum woman treated with either casirivimab/ imdevimab $2.4 \mathrm{~g}$ (patients not hospitalized for COVID-19) or casirivimab/imdevimab $8 \mathrm{~g}$ (patients hospitalized for COVID-19). Patients treated at our outpatient service were sent by general physicians or other territorial medical units dedicated to the follow-up of COVID-19 patients at home. Women admitted to the hospital were treated in different Units of the Careggi University Hospital, Florence, Italy.

\section{Data collection}

Data were captured from the electronic records, including age and gestational age at the time of admission, patient class (inpatient or outpatient), pregnancy class (pregnant or postpartum at administration), gestational outcome (concluded pregnancy, complicated delivery, pregnancy in progress), NIH severity scale at entrance [14], presence of adverse reaction to $\mathrm{mAbs}$ administration, Body Mass Index (BMI), presence of comorbidities. We also collected data on therapeutic strategies used to treat COVID-19 in inpatients.

Data were collected in an Excel spreadsheet (Microsoft, Redmond, CA). Categorical variables are presented as mean and percentages, continuous variables are presented as medians and interquartile ranges. In accordance with the procedure set by the AIFA, patients have been called back after 28 days for a telephonic follow-up.

\section{Results}

\section{Demographics}

A total of 10 patients were treated with mAbs at our hospital in the study period. Two patients were managed in the outpatient clinic, whereas 8 patients were hospitalized (inpatients). The median age was 31 years (IQR 30-33.5, range 29-38). At the time of admission, the mean gestational age was 24 weeks (IQR 21.5-25, range 17-38). Eight were treated during pregnancy and two, admitted to the hospital at 37 and 38 weeks of pregnancy respectively, were treated after delivery.

\section{Clinical characteristics}

No patients were vaccinated for SARS-CoV-2. The median BMI was $24.8 \mathrm{~kg} / \mathrm{m}^{2}$ (IQR 21.62-27.62, range 21.3-30.5). The median number of comorbidities was 1 (IQR $0.25-1$, range $0-3)$. The most prevalent comorbidity was weight excess defined by a BMI $>25 \mathrm{~kg} / \mathrm{m}^{2}$ (4 patients). Two patients were obese $\left(\mathrm{BMI}>30 \mathrm{~kg} / \mathrm{m}^{2}\right)$. All patients presented at least one COVID-19 related symptom, six patients had moderate COVID-19 at the time of mAbs administration, four had severe disease. Variant data were available for nine of the ten patients, and they all were deemed to have the B.1.617.2 variant. 


\section{Maternal treatments and outcomes}

All the patients received casirivimab/imdevimab. Two outpatients and three inpatients with mild symptoms hospitalized for reasons unrelated to COVID-19 (two were hospitalized for giving birth and found to be SARS-CoV-2 positive, one was hospitalized for severe stypsis) received the $2.4 \mathrm{~g}$ dose treatment. Five inpatients seronegative for anti-Spike $\mathrm{IgG}$ at admission received the $8 \mathrm{~g}$ dosage. The treatment was started after a median of 2 days from symptoms onset (range 2-11, IQR 2.25-6.25).

No patient had adverse effects related to the use of mAbs. Only symptomatic treatment with ibuprofen was recommended for outpatients. These patients quickly recovered without any complications.

All inpatients received antithrombotic prophylaxis with enoxaparin for the duration of admission. Among inpatients, four required low flow oxygen via mask/nasal cannulae. Three inpatients were treated with steroids (two received dexamethasone $6 \mathrm{mg} / \mathrm{die}$, one 6-methylprednisolone $1 \mathrm{mg} /$ $\mathrm{kg}$ ) in addition to $\mathrm{mAbs}$. None of the patients received remdesivir. According to the Italian recommendations, seven patients were not eligible for remdesivir treatment ( 6 did not require oxygen therapy and 1 presented more than 10 days after the onset of symptoms). For the remaining three patients this treatment was not considered by the managing physician. One woman (Table 1, patient 7) hospitalized to give birth was treated with $2.4 \mathrm{~g}$ casirivimab/imdevimab. She developed a severe respiratory failure, requiring a transient escalation to high flow oxygen and non-invasive ventilation, but was finally discharged without oxygen support. One patient developed myocarditis, attributed to COVID19 disease complication. No patient required intensive care unit (ICU) admission, and none died. All hospitalized patients were discharged without oxygen support. Among the eight inpatients, the median hospitalization duration was 7 days (range 4-10, IQR 4.75-9.25).

At a 28 days follow-up telephone call, all patients were clinically recovered, and seven patients had performed a negative molecular nasopharyngeal swab for SARS-CoV-2.

\section{Neonatal/fetal outcomes}

At the time of this writing, all women included in the study have already delivered. Five patients had uncomplicated deliveries. Two women had a premature delivery, one because of premature rupture of membranes premature at $34^{+4}$ weeks of pregnancy, one because of pre-eclampsia at 36 weeks of pregnancy requiring urgent caesarean section which was complicated by bleeding and anemia. Another woman required caesarean section because of fetal pathological cardiotocographic trace; however, the neonate was asymptomatic. One woman presented with mild postpartum bleeding. One newborn required transitory admission in the pediatric ICU and another developed mild and transient neonatal jaundice. None of the above events were deemed related to the mAbs treatment considering the timing of mAbs administration and the onset of the event. All women were allowed to breastfeed.

Table 1 summarizes the information on the study patients.

\section{Discussion}

Recently some reports on pregnant women treated with $\mathrm{mAbs}$ have become available, the majority of whom do not report concerning adverse effect and good efficacy [23-27]. This is not a surprise, as immunoglobulins in pregnancy have been largely used to prevent haemolyses caused by maternal/foetal RhD-incompatibility [28]. Moreover, several other monoclonal antibodies are used in pregnant patients [29]. For instance, the NIH guidelines allowed the use of tocilizumab in pregnant patients, stating that pregnant patients with COVID-19 should be treated with the same strategies used for other patients [14]. Our report has several limitations: firstly, we included both patients treated during pregnancy and patients treated in the postpartum. In fact, we considered that patients in the very first days following delivery were still at increased risk of developing severe COVID19, since the vulnerability induced by pregnancy do not immediately recede after delivery $[4,5,15]$. Of note, the patient who required the highest level of care was among those treated after delivery.

Our patient population was demographically similar to other cohorts of pregnant patients with COVID-19 [19]. Moreover, consistently with the epidemiological scenario at the time of mAb administration [30] most of our patients presented with the B.1.617.2 variant. Other studies have also noted how pregnant patients with COVID-19 tend to be relatively healthy [31], which supported the role of pregnancy as a predisposing factor for SARS-CoV-2 infection and severe disease manifestations. Recent advances in pregnancy immunology suggest that pregnancy begins in a pro-inflammatory environment that allows implantation and placentation, then shifts to an anti-inflammatory stage that allows fetal growth, and finally shifts back to a pro-inflammatory stage that promotes labour [32]. The immunological basis underlying pregnant women predisposition to severe COVID-19 is not fully clear. Some authors suggested that the pregnancy induced modifications in Th17 regulationa critical feat of immunomodulation [33] — which could be the basis for an increased inflammatory response to SARS$\mathrm{CoV}-2$ infection in these patients.

Moreover, while Italian recommendations on the use of mAbs do not explicitly list pregnancy as a risk factor for severe COVID-19, they include secondary 


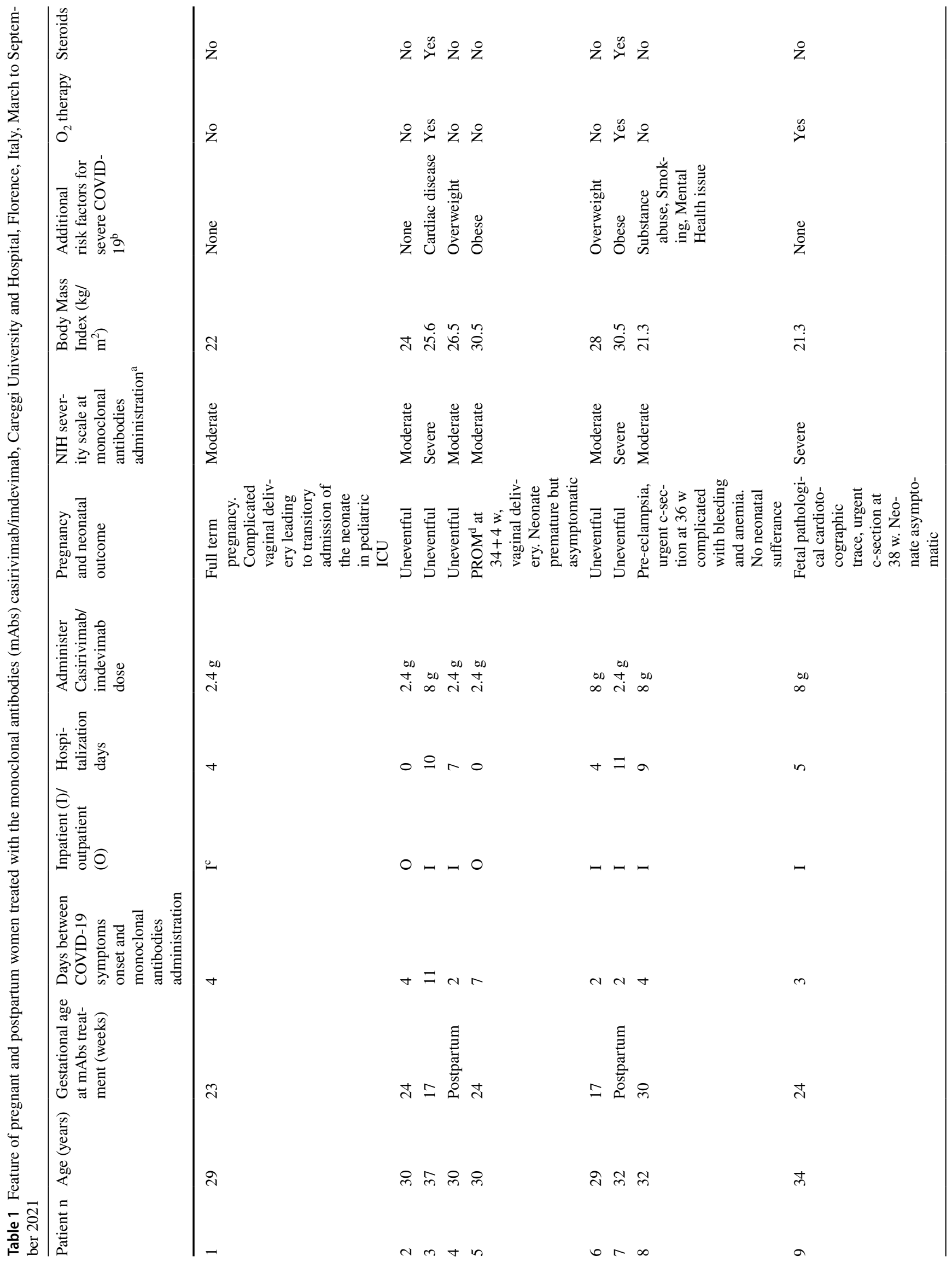




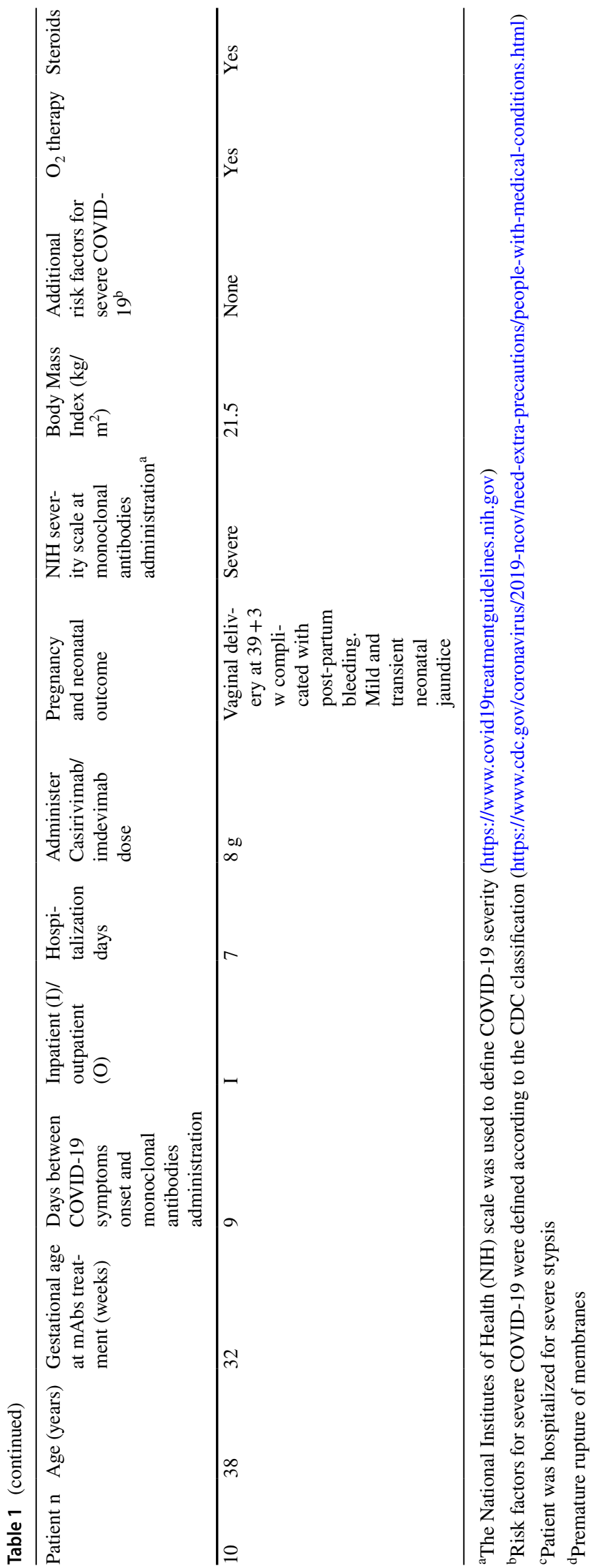

immunodepression as eligibility criteria for $\mathrm{mAbs}$ in the outpatient setting, allowing the use of casirivimab/imdevimab in pregnant patients, as also suggested by the NIH recommendations [14].

Of course, the small sample of patients and the lack of a control group in our study do not make general conclusions about the efficacy and safety of mAbs against SARSCoV-2 in this special population. However, we found that after mAbs administration, most patients did not progress to a severe disease. This is particularly relevant as two meta-analyses showed that the use of maternal ventilation (including C-PAP or mechanical ventilation) worsened the prognosis for both mother and foetus and increased the incidence of pre-term delivery in mother with COVID-19 [6, 34]. Moreover, the use of mAbs does not exclude the use of other drugs currently employed as standard treatment for COVID-19. Some patients in our cohort were treated with steroids due to the presence of pneumonia and hypoxia, like in the RECOVERY trial arms that tested the use of casirivimab/imdevimab $8 \mathrm{gr}$ on inpatients [12].

On the other hand, our experience with outpatients also suggests that casirivimab/imdevimab can be safely used in pregnant inpatients in the early phase of disease since we did not observe mAbs related side effects.

The current lack of data should prompt other authors to share their experience with these compounds in pregnant women. This population is often neglected in clinical studies due to the risk connected with testing new preventive measures or treatment on pregnant women: this once again became true when COVID-19 vaccine trials were conducted $[8,9]$, leading to a delay in the start of immunization campaigns for pregnant women. This is even more important since recent data indicate that the risk for severe COVID-19 increases with gestational age [35].

During the editorial process for this paper, the rise and spread of the Omicron variant of concern has likely rendered the compound used in this case series useless for patients with COVID-19 in areas where this variant is dominant due to the high rate of mutations present in the receptorbinding domain [36]. For this reason, the NIH guidelines were updated to recommend against the administration of casirivimab/imdevimab if Omicron is found to be circulating at a prevalence of over $80 \%$ [14]. Another mAb, sotrovimab, seems to retain efficacy in vitro, but further studies are needed to clarify this in a real life setting [36]. Recently two oral drugs (molnupiravir and nirmatrelvir/ritonavir) became available to treat mild to moderate COVID-19 and data on 3-day early treatment with intravenous remdesivir were published. These drugs are expected to retain activity against the Omicron variant [14]. However, because remdesivir requires intravenous infusion for 3 consecutive days, there may be logistical constraints to administering it in many settings and the oral drugs are not recommended during pregnancy and 
breastfeeding. For this reason, in the "Omicron era" pregnant and postpartum women could become a target population that may benefit from last generation mAbs, such as sotrovimab, since oral treatment options are contraindicated.

In conclusion, our experience suggests that $\mathrm{mAbs}$ against SARS-CoV-2 can be a safe tool to treat pregnant and postpartum patients in the first phases of the disease, or seronegative patients hospitalized for COVID-19. Further studies are needed to assess better the magnitude of the impact on patient morbidity and mortality.

Funding Open access funding provided by Università degli Studi di Firenze within the CRUI-CARE Agreement.

\section{Declarations}

Conflict of interest All authors declare no conflict of interest.

Ethics statement Data collection was approved by the local Ethics Committee (protocol n. 17104_oss). The study was performed in accordance with the ethical principles of the Declaration of Helsinki and with the International Conference on Harmonization Good Clinical Practice guidelines.

Open Access This article is licensed under a Creative Commons Attribution 4.0 International License, which permits use, sharing, adaptation, distribution and reproduction in any medium or format, as long as you give appropriate credit to the original author(s) and the source, provide a link to the Creative Commons licence, and indicate if changes were made. The images or other third party material in this article are included in the article's Creative Commons licence, unless indicated otherwise in a credit line to the material. If material is not included in the article's Creative Commons licence and your intended use is not permitted by statutory regulation or exceeds the permitted use, you will need to obtain permission directly from the copyright holder. To view a copy of this licence, visit http://creativecommons.org/licenses/by/4.0/.

\section{References}

1. Kim L, Garg S, O'Halloran A, Whitaker M, Pham H, Anderson EJ, et al. Risk factors for intensive care unit admission and inhospital mortality among hospitalized adults identified through the US Coronavirus Disease 2019 (COVID-19)-associated hospitalization surveillance network (COVID-NET). Clin Infect Dis [Internet]. 2021;72:e206-14. https://academic.oup.com/cid/artic le/72/9/e206/5872581

2. Grasselli G, Greco M, Zanella A, Albano G, Antonelli M, Bellani $\mathrm{G}$, et al. Risk factors associated with mortality among patients with COVID-19 in intensive care units in Lombardy, Italy. JAMA Intern Med [Internet]. 2020;180:1345-55. http://www.ncbi.nlm. nih.gov/pubmed/32667669

3. CDC - Centers for Disease Control and Prevention. Science brief: evidence used to update the list of underlying medical conditions associated with higher risk for severe COVID-19 [Internet]. 2021. https://www.cdc.gov/coronavirus/2019-ncov/science/sciencebriefs/underlying-evidence-table.html. Accessed 7 Jan 2022.

4. Knobel R, Takemoto MLS, Nakamura-Pereira M, Menezes MO, Borges VK, Katz L, et al. COVID-19-related deaths among women of reproductive age in Brazil: the burden of postpartum. Int J Gynecol Obstet. 2021;155:101-9.

5. Karimi L, Makvandi S, Vahedian-Azimi A, Sathyapalan T, Sahebkar A. Effect of COVID-19 on mortality of pregnant and postpartum women: a systematic review and meta-analysis. J Pregnancy [Internet]. 2021;2021:8870129. http://www.ncbi.nlm.nih.gov/ pubmed/33728066

6. Allotey J, Stallings E, Bonet M, Yap M, Chatterjee S, Kew T, et al. Clinical manifestations, risk factors, and maternal and perinatal outcomes of coronavirus disease 2019 in pregnancy in pregnancy: living systematic review and meta-analysis. BMJ [Internet]. 2019. https://doi.org/10.1136/bmj.m3320.

7. Royal College of Obstetrics and Gynecologists. Coronavirus ( COVID-19 ) Infection in pregnancy-information for healthcare professionals [Internet]. 2021. https://www.rcog.org.uk/globalasse ts/documents/guidelines/2021-12-06-coronavirus-covid-19-infec tion-in-pregnancy-v14.2.pdf. Accessed 25 Dec 2021.

8. Rasmussen SA, Kelley CF, Horton JP, Jamieson DJ. Coronavirus Disease 2019 (COVID-19) vaccines and pregnancy: what obstetricians need to know. Obstet Gynecol [Internet]. 2021;137:408-14. https://doi.org/10.1097/AOG.0000000000004379.

9. Heath PT, Le Doare K, Khalil A. Inclusion of pregnant women in COVID-19 vaccine development. Lancet Infect Dis [Internet]. 2020;20:1007-8. http://www.ncbi.nlm.nih.gov/pubmed/32795409

10. Bassetti M, Giacobbe DR, Bruzzi P, Barisione E, Centanni S, Castaldo N, et al. Clinical management of adult patients with COVID-19 outside intensive care units: guidelines from the Italian Society of anti-infective therapy (SITA) and the Italian Society of Pulmonology (SIP). Infect Dis Ther [Internet]. 2021. http://www. ncbi.nlm.nih.gov/pubmed/34328629. Accessed 7 Jan 2022.

11. Ministero della Salute. Gazzetta Ufficiale della Repubblica Italiana. Serie Generale. 16 giugno 2021. Ministero della Salute; 2021 p. $2-5$.

12. RECOVERY Collaborative Group. Casirivimab and imdevimab in patients admitted to hospital with COVID-19 (RECOVERY): a randomised, controlled, open-label, platform trial. Lancet. 2022;399:665-76. https://doi.org/10.1016/S0140-6736(22) 00163-5.

13. AIFA. DETERMINA 4 agosto 2021 [Internet]. AIFA; 2021. https://www.gazzettaufficiale.it/atto/serie_generale/caricaDett aglioAtto/originario?atto.dataPubblicazioneGazzetta=2021-08$06 \&$ atto .codiceRedazionale $=21 \mathrm{~A} 04884$ \&elenco30 giorni $=$ false

14. NIH. NIH Covid treatment guidelines [Internet]. 2021. https:// www.covid19treatmentguidelines.nih.gov/. Accessed 22 Oct 2021.

15. Kourtis AP, Read JS, Jamieson DJ. Pregnancy and severity of infection. N Engl J Med. 2014;370:2211-8.

16. Lokken EM, Huebner EM, Taylor GG, Hendrickson S, Vanderhoeven J, Kachikis A, et al. Disease severity, pregnancy outcomes, and maternal deaths among pregnant patients with severe acute respiratory syndrome coronavirus 2 infection in Washington State. Am J Obstet Gynecol [Internet]. 2021;225:77e1-14. https://doi. org/10.1016/j.ajog.2020.12.1221.

17. Metz TD, Clifton RG, Hughes BL, Sandoval G, Saade GR, Grobman WA, et al. Disease severity and perinatal outcomes of pregnant patients with Coronavirus Disease 2019 (COVID-19). Obstet Gynecol. 2021;137:571-80.

18. Kalampokas T, Rapani A, Papageorgiou M, Grigoriadis S, Maziotis E, Anifandis G, Triantafyllidou O, Tzanakaki D, Neofytou S, Bakas P, Simopoulou M, Vlahos N. The current evidence regarding COVID-19 and pregnancy: where are we now and where should we head to next? Viruses. 2021;13:2000. https://doi.org/ $10.3390 / \mathrm{v} 13102000$.

19. Jafari M, Pormohammad A, Sheikh Neshin SA, Ghorbani S, Bose $\mathrm{D}$, Alimohammadi $\mathrm{S}$, et al. Clinical characteristics and outcomes of pregnant women with COVID-19 and comparison with control 
patients: a systematic review and meta-analysis. Rev Med Virol. 2021;31:1-16.

20. Collin J, Byström E, Carnahan AS, Ahrne M. Public Health Agency of Sweden's Brief Report: pregnant and postpartum women with severe acute respiratory syndrome coronavirus 2 infection in intensive care in Sweden. Acta Obstet Gynecol Scand. 2020;99:819-22.

21. Villar J, Ariff S, Gunier RB, Thiruvengadam R, Rauch S, Kholin A, et al. Maternal and neonatal morbidity and mortality among pregnant women with and without COVID-19 Infection: the INTERCOVID Multinational Cohort Study. JAMA Pediatr. 2021;175:817-26.

22. Royal College of Obstetrics and Gynaecologists, Royal College of Midwives. Coronavirus (COVID-19) Infection in Pregnancy. Version 14.2: updated Monday 6 December 2021. Guidance for healthcare professionals on coronavirus (COVID-19) infection in pregnancy, including guidance on vaccination in pregnancy, published by the RCOG, Roy [Internet]. 2021. https://www.rcog.org. uk/globalassets/documents/guidelines/2021-12-06-coronaviruscovid-19-infection-in-pregnancy-v14.2.pdf. Accessed 7 Jan 2022.

23. Mayer C, VanHise K, Caskey R, Naqvi M, Burwick RM. Monoclonal antibodies casirivimab and imdevimab in pregnancy for coronavirus disease 2019 (COVID-19). Obstet Gynecol [Internet]. 2021. https://doi.org/10.1097/AOG.0000000000004603.

24. Hirshberg JS, Cooke E, Oakes MC, Odibo AO, Raghuraman N, Kelly JC. Monoclonal antibody treatment of symptomatic COVID-19 in pregnancy: initial report. Am J Obstet Gynecol [Internet]. 2021. https://doi.org/10.1016/j.ajog.2021.08.025.

25. Richley M, Rao RR, Afshar Y, Mei J, Mok T, Vijayan T, Weinstein S, Pham CU, Madamba J, Shin CS, Suda D, Han CS. Neutralizing monoclonal antibodies for Coronavirus Disease 2019 (COVID19) in pregnancy: a case series. Obstet Gynecol. 2022;139:36872. https://doi.org/10.1097/AOG.0000000000004689.

26. Thilagar BP, Ghosh AK, Nguyen J, Theiler RN, Wick MJ, Hurt RT, Razonable RR, Ganesh R. Anti-spike monoclonal antibody therapy in pregnant women with mild-to-moderate Coronavirus Disease 2019 (COVID-19). Obstet Gynecol. 2022. https:// doi.org/10.1097/AOG.0000000000004700.

27. Mayer C, VanHise K, Caskey R, Naqvi M, Burwick RM. Monoclonal antibodies casirivimab and imdevimab in pregnancy for Coronavirus Disease 2019 (COVID-19). Obstet Gynecol.
2021;138:937-9. https://doi.org/10.1097/AOG.0000000000 004603.

28. Urbaniak SJ, Greiss MA. RhD haemolytic disease of the fetus and the newborn. Blood Rev [Internet]. 2000;14:44-61.

29. Hoeltzenbein M, Beck E, Rajwanshi R, Gøtestam Skorpen C, Berber E, Schaefer C, et al. Tocilizumab use in pregnancy: analysis of a global safety database including data from clinical trials and post-marketing data. Semin Arthritis Rheum [Internet]. 2016;46:238-45.

30. ECDC. Variants of concern (VOCs) and variants of interest (VOIs) [Internet]. Wkly. COVID-19 Ctry. Overv. 2021. https:// covid19-country-overviews.ecdc.europa.eu/. Accessed 7 Nov 2021.

31. Bellos I, Pandita A, Panza R. Maternal and perinatal outcomes in pregnant women infected by SARS-CoV-2: a meta-analysis. Eur J Obstet Gynecol Reprod Biol [Internet]. 2021;256:194-204. https://doi.org/10.1016/j.ejogrb.2020.11.038.

32. Mor G, Aldo P, Alvero AB. The unique immunological and microbial aspects of pregnancy. Nat Rev Immunol [Internet]. 2017;17:469-82. https://doi.org/10.1038/nri.2017.64.

33. Cavalcante MB, Cavalcante CTMB, Sarno M, Barini R, KwakKim J. Maternal immune responses and obstetrical outcomes of pregnant women with COVID-19 and possible health risks of offspring. J Reprod Immunol [Internet]. 2021;143:103250. https:// doi.org/10.1016/j.jri.2020.103250.

34. Wei SQ, Bilodeau-Bertrand M, Liu S, Auger N. The impact of COVID-19 on pregnancy outcomes: a systematic review and meta-analysis. CMAJ. 2021;193:E540-8.

35. Badr DA, Picone O, Bevilacqua E, Carlin A, Meli F, Sibiude J, et al. Severe acute respiratory syndrome coronavirus 2 and pregnancy outcomes according to gestational age at time of infection. Emerg Infect Dis. 2021;27:2535-43.

36. Ikemura N, Hoshino A, Higuchi Y, Taminishi S, Inaba T, Matoba S. SARS-CoV-2 Omicron variant escapes neutralization by vaccinated and convalescent sera and therapeutic monoclonal antibodies. 2021;10-4. 